\title{
Postures des scientifiques et interdisciplinarité dans le champ de l'environnement
}

\author{
Evelyne Brun ${ }^{\mathrm{a}}$, Jean-Marie Betsch ${ }^{\mathrm{b}}$, Patrick Blandin ${ }^{\mathrm{c}}$, Geneviève Humbert ${ }^{\mathrm{d}}$, Jean-Claude Lefeuvre ${ }^{\mathrm{e}}$, \\ Marie-Christine Marinval ${ }^{\mathrm{f}}$ \\ a Ingénieur, gestion scientifique, CNRS, Institut d'écologie, biodiversité, évolution, environnement, \\ Université Pierre et Marie Curie, 7 quai Saint-Bernard, 75005 Paris, France \\ b Écologue, MNHN, Département Écologie et gestion de la biodiversité, 57 rue Cuvier, 75005 Paris, France \\ c Écologue, MNHN, Département Hommes, Natures, Sociétés, 57 rue Cuvier, 75005 Paris, France \\ d Juriste, MNHN, Département Écologie et gestion de la biodiversité, 57 rue Cuvier, 75005 Paris, France \\ e Écologue, MNHN, Département Écologie et gestion de la biodiversité, 57 rue Cuvier, 75005 Paris, France \\ f Archéozoologue, Université Paris I, UMR7041 Archéologies et sciences de l'Antiquité, Équipe Archéologie environnementale, \\ Maison de l'archéologie et de l'ethnologie, 21 allée de l’Université, 92023 Nanterre cedex, France
}

L'interrogation sur les pratiques interdisciplinaires s'est souvent portée sur ce qu'apportent les disciplines, mais, à notre connaissance, jamais sur les personnes qui les pratiquent. Parce que chacun d'entre nous ${ }^{1}$, selon le cas, a été à la fois acteur et observateur de la mise en place de l'interdisciplinarité, en tant que chercheur, enseignant, chargé de mission, administrateur de la recherche, président de conseil scientifique, expert, nous avons pris pour objectif de revisiter nos pratiques d'intervention dans ces activités que nous avons convenu de considérer comme des observatoires pour identifier des éléments marquants à partir desquels nous pouvions construire un début de théorisation. Le champ de l'environnement couvrant l'approfondissement des connaissances autant que la demande de gestion et d'action, nous avons été amenés à intégrer dans cette investigation les pratiques disciplinaires qui sont souvent les plus sollicitées. Ce texte présente l'état de notre réflexion après deux années de séminaire et nous souhaitons le mettre en débat.

"Rien ne s'est encore fait par la seule ferveur et par l'attente. Il faut s'y prendre autrement et se mettre à son travail et répondre aux demandes de chaque jour - dans sa vie d'homme, mais aussi dans son métier. »

Max Weber ${ }^{2}$

Auteur correspondant : E. Brun, ebrun@mnhn.fr

${ }^{1}$ Le groupe signataire.

2 Weber, M., 1963. Le Savant et le politique, Paris, Plon.
Dans les années 1970, la Délégation générale à la recherche scientifique et technique (DGRST) tentait, à travers ses programmes, de développer des approches interdisciplinaires pour résoudre des problèmes de déprise agricole. S'appuyant sur l'expérience acquise, dès les années 1980, la recherche française, en s'intéressant au domaine de l'environnement, a proposé d'aborder ces questions en développant une approche systémique et en mettant en œuvre des pratiques interdisciplinaires. Cette démarche a été soutenue par différents établissements publics de recherche et par des ministères. On peut citer les programmes Environnement successifs du CNRS dont, en 1979, le Programme interdisciplinaire de recherche en environnement (PIREN) et, la même année, le nouveau département Systèmes agraires et Développement (SAD) de l'Inra ; enfin, parallèlement, certains programmes du comité Écologie et gestion du patrimoine naturel (EGPN) et, plus tard, le programme Recréer la nature du ministère de l'Environnement.

Les réflexions menées sur la construction de l'interdisciplinarité sont abordées le plus souvent par rapport à (i) ce qu'une discipline peut apporter dans la réflexion sur un sujet, (ii) un objet de recherche, construit à partir d'entrées pertinentes pour plusieurs disciplines, voie mise en avant, notamment dans les Passeurs de frontières ${ }^{3}$.

\footnotetext{
3 Jollivet, M. (Ed.), 1992. Sciences de la nature, sciences de la société : les passeurs de frontières, Paris, CNRS Éditions.
} 
En revanche, il n'y a guère de recherche sur les scientifiques, en tant qu'individus acteurs dans la construction de démarches interdisciplinaires. Notre approche tient pour importante la «posture » du scientifique, celui-ci étant à la fois un «objet » hybride et complexe, biologique, psychique et culturel ${ }^{4}$. Dès lors, on peut s'interroger sur les origines de son regard, de son discours, de ses décisions. Le terme de «posture» nous semble le plus approprié pour définir l'homme dans son activité. Il nous permet d'aborder les manières de penser, d'être et $\mathrm{d}^{\prime}$ agir du scientifique dans ses rapports avec ses environnements professionnels et sociétaux. Nous regarderons le scientifique dans l'activité qu'il exerce, que ce soit la recherche, l'enseignement, l'expertise, la recherche-action, comme un élément interne au système, en l'interrogeant lui plutôt que sa discipline. Dans ces différentes actions, c'est l'homme vecteur d'une idée ou d'une idéologie que nous prendrons en considération, et non pas directement le groupe, la structure ou l'organisation. Il s'agit de montrer que les activités qu'il exerce dépendent de son propre cheminement.

Le scientifique est interpellé de plus en plus sur des questions d'expertise. Notre expérience nous a montré que ce n'est pas un acte banal qui se fonde uniquement sur les concepts et les connaissances développés dans sa discipline, mais qu'il fait intervenir sa façon d'être dans la manière d'aborder la question et d'émettre un avis. Les scientifiques sont-ils préparés à cette fonction?

\section{Le concept de posture du scientifique : quelques jalons}

Nous appellerons "noyau de la posture » la manière d'être du scientifique dans sa dynamique d'interaction avec d'autres personnes, ceci dans une situation professionnelle donnée. Enracinées au plus profond de l'individu, des représentations, des convictions acquises au cours de son histoire et de sa formation, en déterminent les caractéristiques les plus constantes.

Pour le chercheur, c'est le principal moteur qui conduit sa façon d'appréhender et de penser les différentes activités qu'il exerce. La posture s'inscrit dans un système dynamique. La réflexion n'est pas figée, elle évolue au cours du parcours professionnel en fonction de l'acquisition de connaissances nouvelles, de l'expérience, de la maturation des idées en confrontation avec le langage et les apports des autres chercheurs. Il s'agit donc de considérer l'homme dans le processus même de réflexion.

Pour interpréter cette manière d'être, il convient de prendre en compte les origines du scientifique, ses racines culturelles, son milieu social, son éducation, son

\footnotetext{
${ }^{4}$ Morin, E., 1999. Les Sept Savoirs nécessaires à l'éducation du
} futur, Paris, Le Seuil. cursus universitaire, ses opinions politiques, religieuses, son éthique, son sexe. Il faut savoir s'il appartient à différents réseaux professionnels, à des regroupements associatifs. Joue-t-il un rôle dans des ONG ou dans d'autres courants de pensée qui trouvent leur expression dans l'activité de sociétés savantes, voire même occultes?

En passant d'une situation concrète à une autre, l'individu varie différemment, ce qui traduit sa plus ou moins grande capacité d'ajustement au système dans lequel il se trouve interagir. Nous appelons "plasticité du noyau » cette capacité $\mathrm{d}$ 'adaptation à des situations nouvelles. Le concept de noyau recouvre, ainsi, à la fois une résistance au changement et une adaptabilité. Il y a vraisemblablement une gradation de cette plasticité, qui va d'une adaptation provisoire à une situation conjoncturelle jusqu'à une évolution radicale qui provoquera alors un changement de noyau. C'est pourquoi il nous semble important de comprendre l'historique des "empreintes » laissées par certaines influences, généralement extérieures, sur le noyau de la posture.

\section{Le noyau}

Nos observations nous amènent à donner un certain nombre d'exemples où les positions prises traduisent des noyaux fondamentalement différents. Le chercheur se situe-t-il dans ou hors de la nature? Est-il pour une approche systémique ou non? Considère-t-il les systèmes comme plus ou moins en équilibre ou comme intrinsèquement en mouvement? Comment conçoit-il le temps : dans un système en boucle ou cumulatif? La recherche et les sciences répondent-elles à tout? Pense-t-il que la recherche est neutre? Les recherches sur l'environnement doivent-elles être interdisciplinaires? Dans ce domaine, il est fréquent de prendre en compte les actions humaines en les considérant soit comme faisant partie intégrante des systèmes naturels, soit dans les modèles, comme des variables de forçage ${ }^{5}$. De cette posture découlent automatiquement des relations différentes avec le temps, avec l'espace, avec l'approche systémique, avec un type d'interdisciplinarité qui, dans certains cas, peut n'être que de façade.

\section{De la plasticité à l'évolution ou les effets d'empreinte}

Au-delà d'adaptations transitoires et réversibles, un individu peut manifester une évolution. Nous qualifions $\mathrm{d}^{\prime} \ll$ empreinte » le processus par lequel un contexte particulier provoque cette modification. Ce peut être, par exemple dans un cursus, l'allégeance au positionnement

\footnotetext{
5 Terme souvent utilisé par les modélisateurs pour introduire un facteur d'impact humain, par exemple à propos de l'effet de serre additionnel.
} 
scientifique d'un «supérieur » autrefois appelé «Maître». L'empreinte est donc le résultat d'un processus d'imprégnation qui prend place dans l'espace et le temps et qui fait « trace».

La pratique interdisciplinaire peut être révélatrice de ce processus. En effet, elle s'inscrit dans un temps de réflexion nécessaire à la construction d'un objet de recherche commun pertinent, afin de répondre à la question posée. Elle nécessite des négociations pour aboutir à un accord où chacun peut apporter ses compétences dans le respect des concepts et des méthodologies de chacune des disciplines. Selon notre hypothèse, on peut dire que c'est grâce à la plasticité des noyaux des divers partenaires que ce cheminement peut se réaliser.

\section{Retours d'expériences}

Nous étayons notre réflexion sur des exemples qui font apparaître des différences; ces dernières nous permettent d'identifier, dans les processus mis en place par les scientifiques pour aborder les questions posées, ce que nous nommons " posture » et qui, souvent, résulte en partie de l'empreinte du groupe sur l'individu.

\section{Recherches interdisciplinaires, modélisation et posture du modélisateur}

Lorsque nous employons le mot «modèle », nous le faisons en référence à J.-M. Legay ${ }^{6}$, qui rappelle que ce terme peut prendre plusieurs sens : l'objet à imiter, l'exemple, la catégorie, le prototype, la matrice. Comme lui, nous donnons au modèle le statut d'instrument. Le recours à la modélisation est souvent cité par ceux qui pratiquent l'interdisciplinarité comme un outil de dialogue entre disciplines. Cet outil a nécessité des efforts conceptuels importants pour être intégré dans les recherches sur l'environnement, mais des efforts sont encore nécessaires pour prendre en compte les aspects qualitatifs et pas seulement quantitatifs. Souvent, au démarrage d'un projet de recherche, «l'objet » en construction est «modélisé » par des schémas dont la conception est très liée à la discipline du responsable du projet. Aujourd'hui, cela reste vrai même si les modèles multi-agents ou les systèmes d'information géographique ont permis des avancées théoriques et sont très utilisés dans la prise en compte des sociétés humaines. L'intérêt d'un modèle est de permettre de généraliser des observations et des relations identifiées dans un contexte espace/temps particulier ; or, une question environnementale concerne le plus souvent un fait unique dans le temps et dans l'espace. Le

\footnotetext{
${ }^{6}$ Legay, J.-M., 1997. L'Expérience et le modèle : un discours sur la méthode, Paris, INRA Éditions.
}

modèle n'est-il pas, alors, autant révélateur de la posture de son concepteur qu'il est outil pour la recherche? La construction de ces modèles débouche sur une représentation partielle du système étudié. À quoi renvoient les critères sélectionnés? Sont-ils seulement des modes de représentation scientifiques pertinents? Permettent-ils uniquement l'avancement des connaissances ou sont-ils aussi des éléments de négociation entre les disciplines? En quoi le modèle reste-t-il recevable par l'ensemble des chercheurs impliqués dans la réflexion? Lorsque certains chercheurs, utilisateurs des systèmes multi-agents, décident de définir leur activité en l'identifiant comme outil d'accompagnement et de signer une charte ${ }^{7}$, pourquoi ce souci d'identification? Le font-ils parce qu'ils privilégient les questions d'éthique liées à leur travail, parce qu'ils instaurent une sorte de label de qualité ou parce qu'ils veulent que leur méthodologie soit reconnue de la même façon qu'un brevet industriel ? La démarche estelle liée à une pratique de leur institution d'origine et donc révélatrice d'une empreinte?

\section{Les discours sur la « crise » de la biodiversité : révélateurs d'empreintes disciplinaires?}

Face à la «crise » de la biodiversité, même s'il ne semble plus exister de doute sur une accélération de la disparition d'espèces par rapport à des périodes du passé, sommes-nous uniquement dans une démarche scientifique? Pour protéger la planète, faut-il avoir recours au catastrophisme? Pourquoi sommes-nous passés de l'écologie, qui s'appuyait sur les travaux de dynamique de population, de fonctionnement de l'écosystème, d'évolution, etc., à la biodiversité ? Pourquoi ce terme aux multiples définitions est-il devenu un objet de négociations et d'enjeux économiques, alors qu'il fut très longtemps un concept permettant $\mathrm{d}^{\prime}$ 'organiser des recherches sur le risque d'érosion de la diversité du vivant, à plusieurs niveaux? Selon notre conception, une partie de ces questions trouvent leur réponse dans la posture des scientifiques qui ont pu appuyer leur recherche sur une vision fixiste et non pas évolutive de l'écosystème ou d'autres qui ont su alerter la société avec ce concept.

Interrogeons l'état de la biodiversité : selon le scientifique qui s'exprime, les réponses sont différentes.

Pour les systématiciens, l'intérêt premier est d'identifier, et donc de séparer ce qui est semblable de ce qui est différent. Dès les années 1980, l'idée de crise liée à la disparition d'espèces croise les préoccupations de l'UICN et $\mathrm{d}$ 'autres groupes pour aboutir au Sommet Planète Terre à Rio en 1992. La crise ainsi annoncée est investie par la communauté des systématiciens : ceux qui comptent et

\footnotetext{
7 Cf. Collectif ComMod, 2005. La modélisation comme outil d'accompagnement, Natures Sciences Sociétés, 13, 2, 165-168.
} 
qui classent. Certains d'entre eux trouvent une légitimité en participant à des négociations qui débouchent sur des programmes internationaux de recherche ou de gestion des connaissances acquises, tels la création et le développement du Global Biodiversity Information Facility (GBIF), dont l'objectif est de rendre accessibles sur Internet les données de la biodiversité détenues au sein des collections des muséums d'histoire naturelle, des bibliothèques et des banques de données. On peut citer aussi les réunions menées dans le cadre de la Convention sur la diversité biologique $(\mathrm{CDB})^{8}$, dont certaines aboutissent à réglementer l'utilisation des ressources génétiques.

Pour certains écologues qui s'interrogent principalement sur les interactions, la tendance est d'intégrer dans leur réflexion une approche globale du système qu'ils étudient. La même "crise » annoncée est investie par cette communauté qui constate, elle aussi, une accélération du rythme de la disparition d'espèces. Le questionnement développé par ces écologues s'intéresse à la fois aux causes de ces effondrements et aux modifications que cela entraîne dans le fonctionnement et la dynamique de l'écosystème. Tous n'appellent pas ce phénomène une "crise ». Pour eux, la biodiversité est un indicateur de la qualité de l'environnement. C'est pourquoi, dans les années 1995, de nombreux programmes de recherche ont posé la question de l'irréversibilité - ou non - des changements constatés, se sont interrogés sur des questions liées à l'évolution des paysages aussi bien qu'aux divers types de pollution engendrés par les activités humaines et ont débouché sur les aspects santé et environnement. On peut se demander si le principe de précaution n'est pas une norme juridique de protection mise en place à la suite de ces constats 9 .

Lorsque certains économistes et, plus largement, quelques scientifiques en sciences sociales décident d'investir le concept de biodiversité, ils ne se positionnent pas dans une problématique de conservation, mais abordent la question dans une approche liée au développement, avec des actions de gouvernance évoluant vers une notion de développement durable qui, elle, prend en compte la notion de conservation. La question se modifie et devient : en quoi la perte de la biodiversité diminue-telle les services rendus par l'écosystème et se traduit-elle par un coût de remplacement pour la société en place?

Au cours du temps, chaque groupe social, quelles que soient ses origines ou son implantation, a développé un

\footnotetext{
8 La Convention sur la diversité biologique a été signée à Rio de Janeiro le 5 juin 1992 (le texte est disponible sur le site Internet de la CDB : http://www.biodiv.org/doc/legal/cbd-un-fr.pdf). Secrétariat de la Convention sur la diversité biologique : 413 rue Saint-Jacques, Suite 800, Montréal, Québec, Canada H2Y 1 N9.

9 Weill, C., 2003. L'Expertise dans les champs du principe de précaution : propositions, recommandations et commentaires, Paris, IDDRI (document disponible sur Internet : http://www.iddri.org/ iddri/telecharge/notes/03-weill.pdf).
}

classement de ce qu'il nomme «vivant » en fonction de ses propres critères. Le choix qui a été retenu par les systématiciens et accepté par la communauté scientifique dans son ensemble est la classification de Linné (XVIII ${ }^{\mathrm{e}}$ siècle) : une classification hiérarchisée. Elle est aussi une représentation qui oriente la recherche. Aujourd'hui, ce choix est remis en cause par les généticiens.

Mayr écrit, en $1989^{10}$, qu'il n'a pas réussi à trouver dans la littérature une définition convenable du vivant. Curieusement, dans les accords de Rio, il n'a pas été jugé utile d'en donner une. Peut-on dire, alors, qu'il y a une crise de la biodiversité, ou doit-on dire qu'il y a une crise d'une certaine biodiversité : les éléments rares ou menacés, ou celle qui nous est proche, la «nature ordinaire»?

Dans le même temps, il est rassurant que l'homme, grâce à ses capacités d'adaptation et d'extrapolation, mette en place des stratégies utiles à sa survie. Par contre, ne cherche-t-il pas plutôt à dominer la nature parce qu'il a peur du changement pour lui-même, pour sa propre espèce, qu'il ne voudrait plus la voir évoluer, parce qu'il cherche à tout fixer autour de lui (le climat, le temps, les êtres vivants)? Il est légitime de poser cette question, puisque nous sommes dans un monde en perpétuel changement. Comment vivre cette contradiction? Comment déceler, dans les résultats scientifiques, ce qu'a créé la posture du chercheur?

\section{Savoirs scientifiques et savoirs locaux}

La Convention sur la diversité biologique, dans son ensemble, s'intéresse à la conservation des espèces et des milieux. Elle a aussi mis en avant, dans son article 8J, l'importance des savoirs locaux dans ce domaine. La question de la place des savoirs scientifiques face aux savoirs locaux est essentielle et préoccupe depuis longtemps la communauté des sciences sociales. Le savoir peut être défini comme un ensemble de connaissances. Il s'est développé à partir d'observations et de manipulations d'éléments de la nature, pour mieux la comprendre et agir sur elle. Comment distingue-t-on aujourd'hui ces types de savoir?

Le savoir local d'une communauté humaine particulière est solidement ancré dans la pratique, intimement lié à la région dans laquelle cette société s'est développée et adapté aux spécificités de son territoire. Il est ainsi détenu par un groupe de personnes qui ont en commun leur interprétation du monde; il se transmet en général oralement, de génération en génération, en intégrant si nécessaire des innovations. La transmission de ce savoir peut souvent s'apparenter à une empreinte progressive dans un cadre individuel ou collectif. Ce savoir, qualifié

\footnotetext{
10 Mayr, E., 1989. Histoire de la biologie : diversité, évolution et
} hérédité, Paris, Fayard. 
d'empirique par certains scientifiques ${ }^{11}$, résulte d'une représentation de type systémique, construite en prenant en compte un spectre très large de processus et de causes.

Le savoir scientifique se définit par un effort dit «d'objectivation » dans l'étude des phénomènes. Il suppose des observations et des hypothèses testées par des expérimentations. Il se préoccupe du «comment » et aussi du «pourquoi ». Généralement, il est limité à une discipline, c'est donc un savoir partiel. Il est pratiqué par un groupe social particulier, non localisé, appelé «communauté scientifique ». Le savoir « occidental » s'est étendu à la plupart des pays, en imposant ses modes de pensée qui ont laissé une empreinte principalement sur les chercheurs et les enseignants.

Savoirs scientifiques et savoirs locaux n'ont théoriquement pas la même objectivité dans la pensée dominante occidentale. Nous constatons le plus souvent, même lorsque nous pratiquons l'interdisciplinarité, que nous ne nous intéressons qu'à des faits plus ou moins isolés de leur contexte (compréhension des milieux, usages, pratiques, innovation, structure familiale...). Lorsque nous travaillons en partenariat avec les acteurs locaux, nous approchons de la compréhension des choix qui ont été faits à différents niveaux d'intervention et qui se révèlent localement. Bien que ces deux types de savoir, local et scientifique, appréhendent le monde différemment, ils sont tous deux sous l'influence d'une culture et d'une histoire locales.

Dans le contexte d'aujourd'hui, la gestion des problèmes environnementaux nécessite une "rechercheaction », où les scientifiques deviennent des acteurs parmi d'autres, pas moins mais pas plus légitimes que les autres. Nous faisons l'hypothèse que la conduite de telles recherches peut être considérablement influencée par les postures des chercheurs impliqués : leurs conceptions, leurs façons de prendre en compte les «mondes » et les savoirs des autres conditionnent largement la recherche. Le scientifique saura-t-il relativiser son savoir et prendre en compte le «monde » de l'autre et atteindre ainsi une compréhension globale du système étudié?

\section{Le scientifique en situation d'expertise d'un problème d'environnement}

Le scientifique est souvent conduit à produire des expertises. En qualité d'expert, il énonce ce qu'il pense,

\footnotetext{
${ }^{11}$ Cf. Bérard, L., Cegarra, M., Djama, M., Louafi, S., Marchenay, P., Roussel, B., Verdeaux, F. (Eds), 2005. Savoirs et savoir-faire naturalistes locaux : l'originalité française, Paris, IDDRI (disponible sur le site Internet : http://www.iddri.org/iddri/ telecharge/notes/07_savoirs-locaux_fr.pdf). Un ouvrage plus important a été publié l'année suivante par les mêmes auteurs : Biodiversité et savoirs naturalistes locaux en France, Paris, Cirad/IDDRI/IFB/Inra.
}

compte tenu de certains éléments en sa possession provenant d'autres disciplines que la sienne et souvent incomplets ; il n'est plus dans le cadre habituel de la recherche académique et n'a pas à présenter les derniers résultats de ses recherches, à les argumenter et à fournir de nouvelles hypothèses. L'expert est choisi pour une compétence, il doit établir un rapport dans lequel il donne un avis. Quelle légitimité a un chercheur, de la recherche académique ou de la recherche-action, lorsqu'il est choisi comme expert? Le degré de plasticité de sa posture le cantonne-t-il dans une réponse conforme aux questionnements habituels de son «clan » ou l'entraîne-t-il vers l'élargissement de la base de sa réponse ou du champ de recherche à susciter?

Nous distinguons plusieurs types d'expertise qui, selon les cas, obligent ou non à l'interdisciplinarité. Nous pensons que plus la question se complexifie, plus l'expert choisi devra être familiarisé avec la pratique interdisciplinaire. Nous pensons également que c'est grâce à la plasticité de son noyau qu'il sera en capacité d'élaborer des réponses, voire des stratégies, multiples permettant au politique de prendre sa décision.

L'expertise, dans le cadre de la recherche-action et de l'ingénierie, consiste à donner un avis destiné, selon les cas, à éclairer la mise en place de directives, à améliorer la condition humaine avec les hommes concernés, ou même à faire des propositions d'intervention pour ralentir l'évolution d'un système et ainsi laisser à différentes espèces un temps d'adaptation. Appréhender le monde vivant, en tant que système évoluant dans le temps et dans l'espace, conduit le plus souvent à considérer le chercheur comme partie prenante de ce système. De ce fait, son action contribue à l'évolution du système luimême; par la transmission en direct des résultats de la recherche, il devient parfois malgré lui un outil d'aide à la décision.

De plus en plus souvent, le rôle de l'expert scientifique dans les instances internationales tend à s'effacer au profit de l'action des représentants de la société civile au sein d'ONG. Si, dans les années 1990, le choix de l'inscription d'une espèce sur les listes internationales de protection reposait encore sur des avis strictement scientifiques (écologie, biologie des populations), on constate maintenant que ce choix repose aussi désormais sur des critères socio-économico-politiques légitimés par la notion de développement durable et l'influence des grands forums internationaux comme le Sommet de Johannesburg. Face à cette situation, certains experts scientifiques militent au sein d'associations nationales et internationales. En tant que membres de ces associations, et donc de la société civile, ils bénéficient d'une meilleure écoute auprès des décideurs que comme experts, arrivant ainsi à faire prendre en compte certains éléments qui, présentés par un expert scientifique, n'auraient pas été retenus. La stratégie employée révèle une capacité d'adaptation du 
chercheur, qui, confronté à la nécessité de résultat, n'hésite pas à abandonner son statut de scientifique au profit de celui de militant et à disposer ainsi d'un pouvoir pour l'action.

Prenons des exemples où le scientifique est sollicité pour apporter de la connaissance et regardons sa façon de pratiquer l'interdisciplinarité pour y répondre : les deux premiers, suscités par les implications de la législation communautaire et nationale de préservation des milieux naturels ; un troisième, dans le cas d'une « catastrophe naturelle». Comment les postures des chercheurs interviennent-elles dans la prise en compte de la question et, par voie de conséquence, dans la recherche produite?

- Dans le cadre de Natura 2000,1'expertise demandée se fait dans un contexte imposé aux chercheurs par l'application de la directive Habitats ; l'objectif est alors de choisir des sites à partir d'un constat de présence ou d'absence d'espèces. Les experts agissent dans leur champ de compétence et dans leur discipline, sans incitation à l'interdisciplinarité.

- La demande d'expertise pour un projet de parc naturel régional (PNR) ou un schéma d'aménagement et de gestion des eaux (SAGE) a pour objectif de résoudre un problème de gestion compatible avec la réglementation en vigueur. C'est donc d'abord une construction intellectuelle où l'interdisciplinarité peut se développer. Une concertation avec des acteurs locaux est le plus souvent mise en place et des recherches complémentaires peuvent être nécessaires. Les experts doivent s'intégrer dans un travail collectif, avoir une pratique interdisciplinaire; ils peuvent aussi, parfois, par la confrontation avec d'autres chercheurs, bénéficier de l'expérience de projets de même type.

- Dans le cas d'événements exceptionnels tels que la période des crues à Arles, en 2003, on peut supposer que les expertises sont multiples. Ce sont les différents acteurs qui expriment leurs demandes (habitants, aménageurs, pouvoirs publics, assurances, agriculteurs, pêcheurs...). Sur ce territoire, des recherches ont été effectuées par de nombreuses équipes sur des thématiques sociales, physico-chimiques et naturelles. On peut penser qu'une réponse aux expertises demandées, prenant en compte tous ces savoirs, serait de nature à identifier des pistes et à permettre pour un temps d'aboutir à un compromis d'organisation du territoire acceptable par tous. Dans ce cadre-là, les chercheurs, en position d'experts, devraient accepter de mettre leurs connaissances en commun avec d'autres acteurs, en se posant des questions telles que: y a-t-il eu des modifications d'usage de l'environnement? Faut-il abaisser les digues, rendre la liberté au Rhône et inonder la Camargue? Nous pensons que, pour émettre de telles hypothèses, il faut être habitué aux démarches interdisciplinaires et avoir une grande plasticité de posture ; mais nous relevons aussi la difficulté de rester dans le champ strict de l'expertise.

Ces exemples illustrent la diversité des questions qui peuvent être posées au scientifique consulté comme expert. La recherche-action modifie en même temps le système et la société dans laquelle elle intervient. On se trouve alors dans une situation dont les éléments interagissent et changent de façon aléatoire ou chaotique leur trajectoire. Pour le maintenir dans un état stable, si cette demande émane de la société, il devient indispensable d'intervenir pour empêcher son évolution «normale». Les questions posées relèvent tout autant de la protection de la nature que de la gestion du territoire. Les options qui sont prises interfèrent avec la société ; il est donc urgent d'apprendre à appréhender les systèmes complexes et à réfléchir de façon concertée. Sera-t-on en capacité de sortir des sentiers battus? Parce que les choix d'action in fine relèvent $d u$ " politique », le chercheur ne peut privilégier une réponse par rapport à une autre. Où s'arrête la légitimité de l'expert? On peut supposer que son implication reproduira principalement sa posture. Il est donc nécessaire de travailler et de penser autrement, en particulier par la mise en place d'une réelle ingénierie des systèmes naturels et sociaux.

L'évaluation de candidatures ou de réponses à des appels d'offres s'apparente à une expertise dans le cadre de la politique et de la gestion de la recherche. Les chercheurs, experts, sollicités pour rédiger les textes d'appel d'offres montrent souvent de grandes difficultés à exprimer la demande de recherche, bloqués qu'ils sont par leur propre intérêt, intellectuel d'abord, stratégique ensuite pour leur discipline et leur communauté, voire pour leur propre sujet de recherche. Au moment du choix des projets qui seront retenus et donc financés, la question posée, outre la qualité, est l'évaluation de la probabilité d'obtenir les résultats annoncés. Les usages de l'expertise, qui s'appuie le plus souvent sur deux rapporteurs et une discussion au sein d'un comité ad hoc, permettent d'instaurer un dialogue. Les problématiques, les méthodologies, les protocoles, les perspectives de résultats sont largement discutés. Les rapporteurs compétents dans leurs domaines avouent souvent ne pas pouvoir juger de la totalité de la proposition et révèlent ainsi les limites de l'exercice, d'où l'obligation de prendre une décision dans un contexte incertain. Le choix se fait soit en renforçant les certitudes et en même temps l'empreinte de la discipline ou celle du «clan», soit par une ouverture plus ou moins risquée, ouvrant sur un nouveau champ des possibles. Lors de l'évaluation des résultats des recherches, c'est-à-dire en fin de processus, on constate que souvent la personnalité du chef de projet est considérée comme un élément déterminant dans la réussite. On en arrive ainsi à penser qu'il est difficile, lors d'une évaluation se traduisant par une acceptation ou un rejet, d'occulter tant 
la «posture » des auteurs que celle des rapporteurs et des membres du comité.

Nous avons identifié plusieurs types d'expertise, parce qu'elles relèvent d'acteurs et de pratiques différents ; dans les faits, il serait plus juste de dire qu'il s'agit d'une même activité. Les problématiques soumises lors des appels d'offres sont tout autant liées à l'approfondissement des connaissances qu'à une réponse à la demande sociale. Les options prises tout au long de ce processus amènent le chercheur expert à intervenir directement dans la définition des recherches et à devenir inévitablement, par les choix qu'il fait, un acteur; il sort alors malgré lui de la neutralité que la société lui demande de préserver.

\section{Postures des scientifiques et construction de l'interdisciplinarité dans le domaine de l'environnement}

\section{De la nécessité de l'interdisciplinarité}

Les chercheurs ont de plus en plus à répondre à la demande sociale, les questions soulevées mettant l'accent sur la dynamique des processus et des interactions entre matière, espèces, écosystèmes et sociétés humaines. Une démarche, d'abord fondée sur les notions d'équilibre et de stabilité des écosystèmes, a mis progressivement en avant l'idée de la fragilité des systèmes écologiques pour favoriser, à l'opposé, l'idée que les écosystèmes et la biosphère, soumis à des interactions, sont en déséquilibre permanent et en instabilité perpétuelle. Cette prise de conscience a conduit à l'émergence d'une approche associant les sciences de l'homme, les sciences sociales et les sciences de la nature, donc nécessairement interdisciplinaire. Il ne s'agit pas, pour le chercheur, d'embrasser tout seul le problème posé sous tous ses aspects, mais de définir, en collaboration avec des spécialistes des autres sciences, un objet de recherche émergeant du travail collectif ${ }^{12}$, souvent nouveau pour tous les partenaires. Nous sommes persuadés que la construction interdisciplinaire doit prendre en compte bien plus que le mouvement entre les disciplines et que de nombreux blocages observés sont moins liés à ces dernières qu'aux postures des chercheurs impliqués dans la démarche.

\footnotetext{
12 En particulier lors de rencontres organisées par le programme Environnement du CNRS : «Tendances nouvelles en modélisation pour l'environnement. Actes des journées du programme Environnement, vie et sociétés », Paris, Cité des sciences et de l'industrie, 15-17 janvier 1996; "Systèmes écologiques et action de l'homme ", séminaire, Carry-Le-Rouet, 15-17 septembre 1997 ; « Les temps de l'environnement », communications des journées du programme Environnement, vie et sociétés, Toulouse, 5-7 novembre 1997 ; «Systèmes écologiques et action de l'homme », séminaire, Paris, 6- 8 octobre 1999.
}

Qu'il soit dans la recherche théorique ou finalisée, le chercheur ne peut plus se prétendre étranger aux conséquences de l'utilisation des résultats de ses recherches par d'autres. C'est aussi à ce moment-là que, de plus en plus, les décideurs demandent au scientifique de jouer le rôle d'expert. Il est censé être détenteur d'un savoir et de méthodes qui lui donnent la capacité d'évaluer une situation et de faire des propositions au politique, aboutissant à des choix que celui-ci n'ose plus faire. Peut-on encore dire que la recherche est neutre et que le choix du politique n'est pas influencé par le chercheur lui-même?

\section{Un système dominant qui tend vers la normalisation et le formatage}

Le savant encyclopédiste a disparu au profit du scientifique œuvrant dans un domaine de plus en plus restreint. Du fait de la segmentation de l'objet, le scientifique spécialisé est très lié à une technique de plus en plus élaborée, il avance dans la connaissance, mais la vue d'ensemble lui devient étrangère.

Le scientifique exerce son activité au sein de communautés performantes, persuadées qu'elles détiennent la capacité de répondre seules à la plupart des questions qui les concernent, et que celles-ci doivent être simplifiées. La recherche est de plus en plus pointue, s'organise à partir de pôles d'excellence, est élitiste et procède par exclusion. Alors qu'il y a de plus en plus de chercheurs, l'espace dans lequel ils évoluent tend à se restreindre, les filières se multiplient mais deviennent plus étroites.

Par l'évaluation, on choisit le sujet le «meilleur», le plus «pertinent» et le plus « rentable»; les autres sont éliminés et oubliés. Ce processus, permanent, se retrouve dans chaque choix, que ce soit sur des personnes ou sur des projets. Pour avoir des moyens et pour être reconnus, les regroupements entre spécialistes provoquent un isolement et mettent en cause aussi bien l'objectivité des résultats obtenus que la pertinence de la démarche. On est dans un système de "club », de clans ou de réseaux, autant au niveau national qu' international, dans l'organisation de la recherche comme dans celle des publications.

Ce système pousse à la normalisation et au « formatage », il ne fait que renforcer le noyau de la posture et risque d'en réduire la plasticité. L'originalité et l'innovation sont repoussées ; ce mode de fonctionnement supprimant la diversité, on se prive de potentialités. On est dans le disciplinaire très spécialisé qui mène à une structuration en petites unités de spécialistes pointus fonctionnant en circuit fermé. Le chercheur étant souvent attaché à une technique d'investigation, celle-ci peut même devenir le moteur de la démarche à la place de la réflexion et du questionnement. On aboutit alors à un manque de problématique, bien que des résultats obtenus soient publiés dans des revues bien classées, elles aussi très pointues, 
et qui ne sont plus en capacité d'intégrer une approche étrangère à la leur.

La recherche n'est plus explicitée pour la société, mais produite en direction du groupe; ce processus renforce l'effet d'empreinte, permettant au système de perdurer et de s'autoreproduire.

\section{Voir autrement les relations entre scientifiques et sociétés}

Dans le même temps, la pensée complexe et la démarche systémique se structurent dans l'organisation de la recherche par la mise en place de programmes interdisciplinaires. Dans cette démarche qui révèle la posture du chercheur, le fait de penser que l'activité humaine conduit l'humanité à sa perte ou que l'homme est un des éléments de la nature conditionne la manière d'aborder les questions, oriente la construction même de l'objet de recherche et, par voie de conséquence, les résultats.

$\mathrm{Si}$, pour sa carrière, on attend du scientifique qu'il soit toujours plus «pointu» dans son domaine, donc plus spécialisé, il ne peut plus se contenter de l'évaluation par ses pairs, s'il agit dans la recherche-action et l'expertise, qui semblent plutôt exiger de lui une vision globale des problèmes. Il doit désormais se justifier et justifier ses travaux sur les plans éthique et sociétal quant à l'impact qu'ils peuvent avoir sur la société. Si l'on dit que la posture est en partie le résultat de l'empreinte, il est évident, dans l'organisation actuelle de la recherche, que l'originalité et l'innovation ne peuvent émerger qu'à la marge des positions dominantes. Comment amener les scientifiques concernés à travailler sur des questions sociétales?

Les difficultés rencontrées pour pratiquer l'interdisciplinarité sont dues le plus souvent au fait que certains considèrent qu'ils peuvent instrumentaliser les autres disciplines. Ils peuvent même s'improviser compétents dans une discipline distincte de la leur. Quelques-uns s'autoproclament interdisciplinaires à eux seuls. Certains scientifiques dénient la qualité de "scientifique» aux sciences humaines, qualifiées en l'occurrence de «sciences molles ${ }^{13}$ ». C'est peut-être d'abord par ses attitudes face ses collègues qu'un scientifique se dévoile, refermé sur son «monde » ou ouvert sur celui d'autrui.

\section{Conclusion}

On a pu observer qu'à partir d'une même question, les scientifiques prennent des trajectoires différentes pour y répondre et que les mêmes disciplines se retrouvent dans les diverses options choisies. Ce ne sont donc pas les

${ }_{13}$ Mucchelli, L., 2005. Sciences dures et sciences molles, La Recherche, 386, 59-62. disciplines qui expliquent essentiellement la démarche du scientifique. Le concept de posture nous paraît déterminant pour expliciter les choix des scientifiques qui décident de mettre leurs compétences en commun, en particulier au moment de l'élaboration de la question et de la construction de l'objet de la recherche dans la pratique interdisciplinaire. C'est alors que se révéleront leurs représentations et leurs convictions, ainsi que leurs capacités d'ajustement. Il est donc possible d'affirmer que la recherche interdisciplinaire se fera d'autant mieux qu'elle réunira des scientifiques ayant des postures compatibles, capables de convergence.

C'est pourquoi la recherche, l'expertise, l'enseignement ne sont jamais neutres. Il est donc impératif de se donner des modes de fonctionnement, une déontologie, puisqu'on est amené à renforcer une idéologie, que ce soit consciemment ou non. La recherche-action, qu'elle soit liée à l'expertise, à l'aménagement de territoires ou à des mesures de conservation, modifie la nature et, avec elle, la société, localement et globalement, non plus seulement par une évolution lente des pratiques, mais par une réflexion dont les propositions peuvent avoir un impact rapide.

Dans ce contexte, comment organiser les différentes activités qui s'appuient sur une recherche solide et une expertise qui proposent des réponses multiples à la question posée et permettent des choix ouverts sur des possibles et non pas des réponses attendues? En 1997, Roqueplo proposait déjà l'institutionnalisation de procédures d'expertise comportant un "espace de la critique scientifique des options envisageables ${ }^{14}$ ». Qu'en est-il aujourd'hui?

Le recours aux expertises est devenu une pratique courante aussi bien pour les gouvernements que pour la société civile. Les différentes conventions internationales s'appuient, elles aussi, sur l'expertise. Celle-ci est la base de décisions politiques impliquant souvent la totalité de la planète. Comment permettre au scientifique d'effectuer sa mission dans un contexte de responsabilité partagée, lui garantissant de pouvoir rendre son rapport, sans pression de la part de quiconque, tout en sachant qu'il introduit forcément un biais? Nous disons qu'il est important de se doter d'une science pour une ingénierie des systèmes naturels et sociaux, afin de prendre en compte le glissement de l'expertise vers une action concrète conduisant à une acceptation sociale négociée.

On peut dire que le scientifique dispose d'outils pour faire aboutir sa recherche et que sa discipline, avec ses méthodes, ses concepts, ses paradigmes, est son outil privilégié. Avec la notion de posture, nous passons d'un chercheur qui utilise des outils, dont sa discipline, à un

\footnotetext{
14 Roqueplo, P., 1997. Entre savoir et décision, l'expertise scientifique, Paris, INRA Éditions.
} 
acteur créateur de son activité. Dans le même temps, les scientifiques, qui se pensaient isolés dans leurs laboratoires, se trouvent face à une nouvelle responsabilité, leur recherche étant de plus en plus étroitement liée à l'évolution des sociétés.

L'importance que nous attribuons à la notion de posture des acteurs de la science dans leur activité, et plus particulièrement dans la recherche interdisciplinaire, nous conduit à poser le problème des interactions entre les scientifiques et d'autres acteurs qui sont appelés à analyser les questions environnementales et à s'interroger sur leur rôle «politique » dans la société.

\section{Remerciements}

Les auteurs tiennent à remercier Robert Barbault et Serge Bahuchet pour avoir permis la tenue du séminaire à l'origine de ce texte, pour leurs conseils et leurs encouragements.

To access this journal online: www.edpsciences.org 